\title{
Exact and Solitary Wave Solutions to the Generalized Fifth-order KdV Equation by Using the Modified Simple Equation Method
}

\author{
M. Ashrafuzzaman Khan, M. Ali Akbar \\ Department of Applied Mathematics, University of Rajshahi, Rajshahi, Bangladesh
}

Email address:

akhanmath@yahoo.com (M. A. Khan),ali_math74@yahoo.com (M. A. Akbar)

To cite this article:

M. Ashrafuzzaman Khan, M. Ali Akbar. Exact and Solitary Wave Solutions to the Generalized Fifth-order KdV Equation by Using the Modified Simple Equation Method. Applied and Computational Mathematics. Vol. 4, No. 3, 2015, pp. 122-129.

doi: 10.11648/j.acm.20150403.14

\begin{abstract}
Although the modified simple equation (MSE) method effectively provides exact traveling wave solutions to nonlinear evolution equations (NLEEs) in the field of engineering and mathematical physics, it has some limitations. When the balance number is greater than one, usually the method does not give any solution. In this article, we have exposed a process how to implement the MSE method to solve NLEEs for balance number two. In order to verify the process, the generalized fifth-order KdV equation has been solved. By means of this scheme, we found some fresh traveling wave solutions to the above mentioned equation. When the parameters receive special values, solitary wave solutions are derived from the exact solutions. We analyze the solitary wave properties by the graphs of the solutions. This shows the validity, usefulness, and necessity of the process.
\end{abstract}

Keywords: MSE Method, Nonlinear Evolution Equations, Solitary Wave Solutions, Exact Solutions,

Generalized Fifth-Order Kdv Equation

\section{Introduction}

Nonlinear evolution equations occur not only from many fields of mathematics, but also from other branches of science such as physics, material science, mechanics etc. Intricacy of NLEEs and challenges in their theoretical study has attracted lots of attention from numerous mathematicians and scientists who are concern with nonlinear sciences. Therefore, the studies of exact solutions to NLEEs play a very important role to know the inner structure of the nonlinear phenomena. But the basic problem is, it is not easy to attain their exact solutions. Therefore, in order to examine exact solutions, different groups of mathematicians and physicist are working jointly. In the recent years, considerable developments have been made for searching exact solutions to NLEEs. They established several methods, such as, the inverse scattering transformation method [1], the Hirota's bilinear method [2], the Backlund transformation method ([3][4]), the Darboux transformation method [5], the Painleve expansion method [6], the Adomian decomposition method ([7][8]), the He's homotopy perturbation method ([9][10]), the Jacobi elliptic function method ([11][12]), the
Miura transformation method [13], the sine-cosine method ([14][15]), the homogeneous balance method [16], the tanhfunction method ([17][18]), the extended tanh-function method ([19] [20]), the first integration method [21], the Fexpansion method [22],the auxiliary equation method [23], the Lie group symmetry method [24], the variational iteration method [25], the ansatz method ([26][27]), the Exp-function method ([28][29]), the $\left(G^{\prime} / G\right)$-expansion method ([30][35]), the modified simple equation method ([36]-[40]), the $\exp (-\phi(\eta))$-expansion method ([41][42]), etc.

The modified simple equation method ([36]-[40]) is a recently developed rising method. Its computation is straightforward, systematic, and no need the symbolic computation software to manipulate the algebraic equations. But, the method has some shortcoming, when the balance number is greater than one, usually the method does not give any solution. To the best of our knowledge, till now only two articles are available in the literature concerning higher balance number (for balance number two). Salam [43] used the MSE method to the modified Liouville equation (wherein the balance number is two) and write-down a solution to this equation. However, unfortunately the obtained solution does 
not satisfy the equation. And in Ref. [44], Zayed and Arnous solved the KP-BBM equation by means of the MSE method and found some solutions of this equation. But there is no guideline in this article, how one can solve other NLEEs for the higher balance number. In the present article, we have developed a technique so that the MSE method can be exploited to solve NLEEs for balance number two. Inserting the assumed solution to the corresponding ordinary differential equation and then equating the coefficients of $s(\xi)^{-j},(j=0,1,2, \cdots, N)$ yields an over-determined set of algebraic and differential equations. During determination the unknown function, there born a third order linear ordinary differential equation in $s$ and $\xi$. If in the solution of $s, \xi$ appear as a polynomial, it will not be eligible to receive as solitary wave solution, because for solitary wave solution, we know that $|u| \rightarrow 0$ as $\xi \rightarrow \pm \infty$ [7]. Therefore, the coefficients of the polynomial must be zero. This constraint is essential to solve NLEEs for higher balance number.

The article is organized as follows: In section 2, we summarize the description of the method. In section 3 , we employ the method to NLEEs with balance number two. In section 4, the physical explanations of the solutions are presented and in section 5, we have drawn our conclusions.

\section{The Method}

Let us consider the nonlinear evolution equation of the form

$$
H\left(u, u_{t}, u_{x}, u_{y}, u_{z}, u_{x x}, u_{t t}, \ldots\right)=0,
$$

where $u=u(x, y, z, t)$ is an unidentified function, $H$ is a polynomial in $u(x, y, z, t)$ and its partial derivatives, which include the highest order derivatives and nonlinear terms of the highest order, and the subscripts denote partial derivatives. In order to solve (2.1) by means of the MSE method [36-40], we have to execute the following steps:

Step 1: The traveling wave variable,

$$
u(x, y, z, t)=u(\xi), \xi=k(x+y+z \pm \omega t)
$$

permits us to transform the Eq. (2.1) into the following ordinary differential equation (ODE):

$$
G\left(u, u^{\prime}, u^{\prime \prime}, \cdots\right)=0
$$

where $G$ is a polynomial in $u(\xi)$ and its derivatives, wherein $u^{\prime}(\xi)=\frac{d u}{d \xi}$.

Step 2:We suppose that the solution of (2.3) can be express in the form,

$$
u(\xi)=\sum_{i=0}^{N} a_{i}\left[\frac{s^{\prime}(\xi)}{s(\xi)}\right]^{i}
$$

where $a_{i},(i=0,1,2, \cdots, N)$ are unknown constants to be determined, such that $a_{N} \neq 0$, and $s(\xi)$ is an unknown function to be evaluated. In tanh-function method, $\left(G^{\prime} / G\right)$ expansion method, sine-cosine method, Jacobi elliptic function method, Exp-function method etc., the solutions are proposed in terms of some functions established in advance, but in the MSE method, $s(\xi)$ is not pre-defined or not a solution of any pre-defined differential equation. Therefore, it is not possible to conjecture from earlier what kind of solutions one may get through this method. This is the individuality and distinction of this method. Therefore, some fresh solutions might be found by this method.

Step 3: The positive integer $N$ appearing in Eq. (2.4) can be determined by taking into account the homogeneous balance between the highest order nonlinear terms and the derivatives of highest order occurring in Eq. (2.3).

Step 4: We substitute (2.4) into (2.3) and then we account the function $s(\xi)$. As a result of this substitution, we get a polynomial of $\left(s^{\prime}(\xi) / s(\xi)\right)$ and its derivatives. In the resultant polynomial, we equate all the coefficients of $(s(\xi))^{-i},(i=0,1,2, \ldots, N)$ to zero. This procedure yields a system of algebraic and differential equations which can be solved for getting $a_{i}(i=0,1,2, \cdots, N), s(\xi)$ and the value of the other needful parameters. This completes the determination of the solutions to the equation (2.1).

\section{Application of the Method}

In this section, we will execute the MSE method to extract solitary wave solutions to the generalized fifth-order $\mathrm{KdV}$ equation which is very important in the fields of surface wave propagation on shallow water surfaces. Let us consider the generalized fifth-order $\mathrm{KdV}$ equation of the form

$$
u_{t}+\alpha u u_{x}+\beta u^{2} u_{x}+\gamma u_{x x x}+\mu u_{x x x x x}=0
$$

where $\alpha, \beta, \gamma$ and $\mu$ are the real constants.

To construct solitary wave solutions of the generalized fifth-order $\mathrm{KdV}$ equation by applying the MSE method, we use the wave variable

$$
u(x, t)=U(\xi), \quad \xi=k(x-\omega t) .
$$

The traveling wave transformation (3.2) reduces Eq. (3.1) to the following ODE in the form:

$$
-\omega U^{\prime}+\alpha U U^{\prime}+\beta U^{2} U^{\prime}+\gamma k^{2} U^{\prime \prime \prime}+\mu k^{4} U^{(v)}=0
$$

where prime denotes the derivatives with respect to $\xi$. Now, integrating the Eq. (3.3) with respect to $\xi$, we get a new ODE in the form

$$
-\omega U+\alpha \frac{U^{2}}{2}+\beta \frac{U^{3}}{3}+\gamma k^{2} U^{\prime \prime}+\mu k^{4} U^{(i v)}=0
$$

Balancing the highest order derivative term $U^{(i v)}$ and the nonlinear term of the highest order $U^{3}$ occurring in (3.4), we 
get $N=2$. Thus, the solution (2.4) takes the form

$$
U(\xi)=a_{0}+a_{1}\left(\frac{s^{\prime}}{s}\right)+a_{2}\left(\frac{s^{\prime}}{s}\right)^{2} .
$$

where $a_{0}, a_{1}$ and $a_{2}$ are constants to be determined, such that $a_{2} \neq 0$, and $s(\xi)$ is an unknown function to be determined. Now, it is easy to accomplish

$$
\begin{aligned}
& U^{\prime}=-\frac{a_{1}\left(s^{\prime}\right)^{2}}{s^{2}}-\frac{2 a_{2}\left(s^{\prime}\right)^{3}}{s^{3}}+\frac{a_{1} s^{\prime \prime}}{s}+\frac{2 a_{2} s^{\prime} s^{\prime \prime}}{s^{2}} . \\
& U^{\prime \prime}=\frac{2 a_{1}\left(s^{\prime}\right)^{3}}{s^{3}}+\frac{6 a_{2}\left(s^{\prime}\right)^{4}}{s^{4}}-\frac{3 a_{1} s^{\prime} s^{\prime \prime}}{s^{2}}-\frac{10 a_{2}\left(s^{\prime}\right)^{2} s^{\prime \prime}}{s^{3}} \\
& +\frac{2 a_{2}\left(s^{\prime \prime}\right)^{2}}{s^{2}}+\frac{a_{1} s^{\prime \prime \prime}}{s}+\frac{2 a_{2} s^{\prime} s^{\prime \prime \prime}}{s^{2}} \\
& U^{\prime \prime \prime}=\frac{1}{s^{5}}\left[2 a _ { 2 } \left\{-12\left(s^{\prime}\right)^{5}+27 s\left(s^{\prime}\right)^{3} s^{\prime \prime}+3 s^{3} s^{\prime \prime} s^{\prime \prime \prime}\right.\right. \\
& \left.-s^{2} s^{\prime}\left(7 s^{\prime} s^{\prime \prime}+12\left(s^{\prime \prime}\right)^{2}-s s^{(i v)}\right)\right\}-6 a_{1} s\left(s^{\prime}\right)^{4} \text {. } \\
& \left.+a_{1} s^{2}\left\{12\left(s^{\prime}\right)^{2} s^{\prime \prime}-s\left(4 s^{\prime} s^{\prime \prime \prime}+3\left(s^{\prime \prime}\right)^{2}-s s^{(i v)}\right)\right\}\right] \\
& U^{(i v)}=\frac{a_{1}}{s^{5}}\left\{24\left(s^{\prime}\right)^{5}-60 s\left(s^{\prime}\right)^{3} s^{\prime \prime}+20 s^{2}\left(s^{\prime}\right)^{2} s^{\prime \prime \prime}\right. \\
& -5 s^{2} s^{\prime}\left(-6\left(s^{\prime \prime}\right)^{2}+s s^{(i v)}\right)+s^{3}\left(-10 s^{\prime \prime} s^{\prime \prime \prime}\right. \\
& \left.\left.+s s^{(v)}\right)\right\}+\frac{2 a_{2}}{s^{6}}\left[60\left(s^{\prime}\right)^{6}-168 s\left(s^{\prime}\right)^{4} s^{\prime \prime}\right. \text {. } \\
& +3 s^{2}\left(s^{\prime}\right)^{2}\left\{16 s^{\prime} s^{\prime \prime \prime}+3\left(13\left(s^{\prime \prime}\right)^{2}-s s^{(i v)}\right)\right\} \\
& +s^{3}\left\{-12\left(s^{\prime \prime}\right)^{3}+3 s\left(s^{\prime \prime \prime}\right)^{2}+4 s s^{\prime \prime} s^{(i v)}\right. \\
& \left.\left.+s^{\prime}\left(-44 s^{\prime \prime} s^{\prime \prime \prime}+s s^{(v)}\right)\right\}\right]
\end{aligned}
$$

Substituting the values of $U, U^{\prime}, U^{\prime \prime}, U^{\prime \prime \prime}$ and $U^{(i v)}$ from (3.5)-(3.9) into Eq. (3.4) and then equating the coefficients of $s^{0}, s^{-1}, s^{-2}, s^{-3}, s^{-4}$ to zero, we respectively obtain

$$
\left.\begin{array}{c}
\frac{a_{0}}{6}\left(-6 \omega+3 \alpha a_{0}+2 \beta a_{0}^{2}\right)=0 . \\
a_{1}\left\{\left(-\omega+\alpha a_{0}+\beta a_{0}^{2}\right) s^{\prime}+k^{2} \gamma s^{\prime \prime \prime}+k^{4} \mu s^{(v)}\right\}=0 . \\
\frac{1}{2}\left(\alpha+2 \beta a_{0}\right) a_{1}^{2}\left(s^{\prime}\right)^{2}+2 a_{2} k^{2} \gamma\left\{4 k^{2} s^{\prime \prime} s^{(i v)}\right. \\
\left.+\left(s^{\prime \prime}\right)^{2}+3 k^{2}\left(s^{\prime \prime \prime}\right)^{2}+s^{\prime}\left(s^{\prime \prime \prime}+k^{2} s^{(v)}\right)\right\} \\
-a_{1} k^{2} \gamma\left\{10 k^{2} s^{\prime \prime} s^{\prime \prime \prime}+s^{\prime}\left(3 s^{\prime \prime}+5 k^{4} s^{(i v)}\right)\right\} \\
+a_{2}\left(-\omega+\alpha a_{0}+\beta a_{0}^{2}\right)\left(s^{\prime}\right)^{2}
\end{array}\right]=0 .
$$

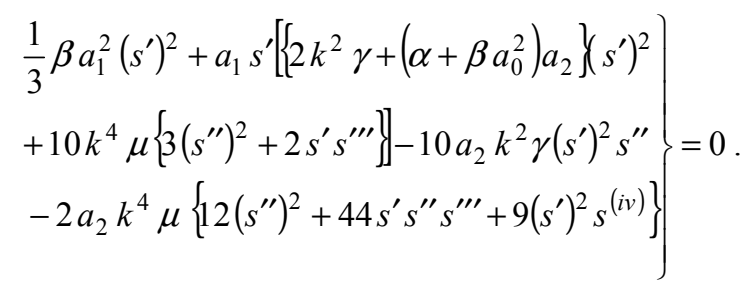

$$
\left.\begin{array}{c}
\frac{1}{2}\left(\alpha+2 \beta a_{0}\right) a_{2}^{2}\left(s^{\prime}\right)^{4}-60 a_{1} k^{4} \mu\left(s^{\prime}\right)^{2} s^{\prime \prime} \\
+2 k^{4} \mu a_{2}\left(s^{\prime}\right)^{2}\left\{117\left(s^{\prime \prime}\right)^{2}+48 s^{\prime} s^{\prime \prime \prime}\right\} \\
+a_{2}\left(6 k^{2} \gamma+\beta a_{1}^{2}\right)\left(s^{\prime}\right)^{4}
\end{array}\right]=0 .
$$

$\left\{a_{1}\left(24 k^{4} \mu+\beta a_{2}^{2}\right)-336 k^{4} \mu a_{2} s^{\prime \prime}\right\}\left(s^{\prime}\right)^{4}=0$.

$$
\frac{1}{3} a_{2}\left(360 k^{4} \mu+\beta a_{2}^{2}\right)\left(s^{\prime}\right)^{6}=0 .
$$

From Eqs. (3.10) and (3.16), we obtain

$$
a_{0}=0 \text { and } a_{2}= \pm \frac{i 6 \sqrt{10} k^{2} \sqrt{\mu}}{\sqrt{\beta}} \text {, since } a_{2} \neq 0 .
$$

Therefore, the following cases arise depending on the values of $a_{2}$.

Case 1: When $a_{2}=\frac{6 i \sqrt{10} k^{2} \sqrt{\mu}}{\sqrt{\beta}}$, then from Eqs. (3.11)(3.15), we obtain

$$
\begin{gathered}
a_{1}= \pm 6 \sqrt{\frac{2 k^{2} \gamma}{\beta}+\frac{i \sqrt{10} k^{2} \alpha \sqrt{\mu}}{\beta^{3 / 2}}}, \\
\omega=-\frac{8 \beta \gamma^{2}+3 i \sqrt{10} \alpha \sqrt{\beta} \gamma \sqrt{\mu}+5 \alpha^{2} \mu}{50 \beta \mu},
\end{gathered}
$$

and

$$
s(\xi)=\frac{\mp i \sqrt{10} k \beta^{1 / 4} \sqrt{\mu} c_{1}}{\sqrt{2 \sqrt{\beta} \gamma+i \sqrt{10} \alpha \sqrt{\mu}}} e^{ \pm i \xi \frac{\sqrt{2 \sqrt{\beta} \gamma+i \sqrt{10} \alpha \sqrt{\mu}}}{\sqrt{10} k \beta^{1 / 4} \sqrt{\mu}}}+c_{2} .
$$

where $c_{1}$ and $c_{2}$ are integrating constants.

Now, substituting the values of $a_{0}, a_{1}, a_{2}$ and $s(\xi)$ into Eq. (3.5), we obtain 


$$
U(\xi)= \pm \frac{6 k(2 \sqrt{\beta} \gamma+i \sqrt{10} \alpha \sqrt{\mu})^{3 / 2} \beta^{-3 / 4} c_{1} c_{2} e^{ \pm i \xi \frac{\sqrt{2 \sqrt{\beta} \gamma+i \sqrt{10} \alpha \sqrt{\mu}}}{\sqrt{10} k \beta^{1 / 4} \sqrt{\mu}}}}{\left(c_{2} \sqrt{2 \sqrt{\beta} \gamma+i \sqrt{10} \alpha \sqrt{\mu}} \mp i \sqrt{10} k \beta^{1 / 4} \sqrt{\mu} c_{1} e^{ \pm i \xi \frac{\xi \sqrt{2 \sqrt{\beta} \gamma+i \sqrt{10} \alpha \sqrt{\mu}}}{\sqrt{10} k \beta^{1 / 4} \sqrt{\mu}}}\right)^{2}} .
$$

Simplifying the required solution (3.17), we obtain the following close-form solution to the generalized fifth-order KdV equation (3.1):

$$
u(x, t)=\mp \frac{6 k \beta^{-3 / 4}(2 \sqrt{\beta} \gamma+i \sqrt{10} \alpha \sqrt{\mu})^{3 / 2} c_{1} c_{2}}{\left\{\sqrt{10} k \beta^{1 / 4} \sqrt{\mu} c_{1}(\cos (\theta) \pm i \sin (\theta)) \pm i c_{2} \sqrt{2 \sqrt{\beta}} \gamma+i \sqrt{10} \alpha \sqrt{\mu}(\cos (\theta) \mp i \sin (\theta))\right\}^{2}} .
$$

Where

$$
\theta=\frac{50 \sqrt{10} \beta \mu x+\left(8 \sqrt{10} \beta \gamma^{2}+30 i \alpha \sqrt{\beta} \gamma \sqrt{\mu}+5 \sqrt{10} \alpha^{2} \mu\right) t}{1000 \beta^{5 / 4} \mu^{3 / 2}(2 \sqrt{\beta} \gamma+i \sqrt{10} \alpha \sqrt{\mu})^{-1 / 2}}
$$

Since $c_{1}$ and $c_{2}$ are arbitrary constants, one may randomly pick their values. If we choose $c_{1}=\sqrt{2 \sqrt{\beta} \gamma+i \sqrt{10} \alpha \sqrt{\mu}}$ and $c_{2}=-\sqrt{10} k \beta^{1 / 4} \sqrt{\mu}$ then from (3.18), we obtain the following solitary wave solution:

$$
u_{1}(x, t)=\frac{3\left(2 \sqrt{10} \beta \gamma^{2}+i 20 \alpha \sqrt{\beta} \gamma \sqrt{\mu}-5 \sqrt{10} \alpha^{2} \mu\right)}{5 \beta(\sqrt{10} \alpha \sqrt{\mu}-i 2 \sqrt{\beta} \gamma) \sqrt{\mu}\left\{1-\sin \left(\frac{\theta}{2}\right)\right\}} .
$$

Again, if we choose $c_{1}=\sqrt{2 \sqrt{\beta} \gamma+i \sqrt{10} \alpha \sqrt{\mu}}$ and $c_{2}=\sqrt{10} k \beta^{1 / 4} \sqrt{\mu}$ then from (3.18), we obtain

$$
u_{2}(x, t)=\frac{3\left(2 \sqrt{10} \beta \gamma^{2}+i 20 \alpha \sqrt{\beta} \gamma \sqrt{\mu}-5 \sqrt{10} \alpha^{2} \mu\right)}{5 \beta(\sqrt{10} \alpha \sqrt{\mu}-i 2 \sqrt{\beta} \gamma) \sqrt{\mu}\left\{1+\sin \left(\frac{\theta}{2}\right)\right\}}
$$

On the other hand, if $c_{1}=\sqrt{2 \sqrt{\beta} \gamma+i \sqrt{10} \alpha \sqrt{\mu}}$ and $c_{2}= \pm i \sqrt{10} k \beta^{1 / 4} \sqrt{\mu}$, from solution (3.18), we derive the solitary wave solutions in the form:

$$
u_{3}(x, t)=-\frac{3(-i \sqrt{10} \sqrt{\beta} \gamma+5 \alpha \sqrt{\mu})}{5 \beta \sqrt{\mu}\left\{-1+\cos \left(\frac{\theta}{2}\right)\right\}} .
$$

Also when $\quad c_{1}=\sqrt{2 \sqrt{\beta}} \gamma+i \sqrt{10} \alpha \sqrt{\mu} \quad$ and $c_{2}=\mp i \sqrt{10} k \beta^{1 / 4} \sqrt{\mu}$ then from solution (3.18) can be written as the following solitary wave solutions in the form:

$$
u_{4}(x, t)=-\frac{3(-i \sqrt{10} \sqrt{\beta} \gamma+5 \alpha \sqrt{\mu})}{5 \beta \sqrt{\mu}\left\{1+\cos \left(\frac{\theta}{2}\right)\right\}} .
$$

Case 2: When $a_{2}=-\frac{6 i \sqrt{10} k^{2} \sqrt{\mu}}{\sqrt{\beta}}$, then from Eqs. (3.11)-(3.15), we get

$$
\begin{gathered}
a_{1}= \pm \frac{6 k \sqrt{2 \sqrt{\beta} \gamma-i \sqrt{10} \alpha \sqrt{\mu}}}{\beta^{3 / 4}}, \\
\omega=-\frac{8 \beta \gamma^{2}-i 3 \sqrt{10} \alpha \sqrt{\beta} \gamma \sqrt{\mu}+5 \alpha^{2} \mu}{50 \beta \mu}
\end{gathered}
$$

And

$$
\begin{gathered}
s(\xi)= \pm \frac{i \sqrt{10} k \beta^{1 / 4} \sqrt{\mu} c_{1}}{\sqrt{2 \sqrt{\beta} \gamma-i \sqrt{10} \alpha \sqrt{\mu}}} e^{\mp i \xi \frac{\sqrt{2 \sqrt{\beta} \gamma-i \sqrt{10} \alpha \sqrt{\mu}}}{\sqrt{10} k \beta^{1 / 4} \sqrt{\mu}}}+c_{2} \cdot \mathrm{W} \\
\text { here } c_{1} \text { and } c_{2} \text { are integrating constants. }
\end{gathered}
$$

Now, substituting the values of $a_{0}, a_{1}, a_{2}$ and $s(\xi)$ into Eq. (3.5), we obtain the solution in the form: 


$$
U(\xi)= \pm \frac{6 k(2 \sqrt{\beta} \gamma-i \sqrt{10} \alpha \sqrt{\mu})^{3 / 2} \beta^{-3 / 4} c_{1} c_{2} e^{ \pm i \xi \frac{\sqrt{2 \sqrt{\beta} \gamma-i \sqrt{10} \alpha \sqrt{\mu}}}{\sqrt{10} k \beta^{1 / 4} \sqrt{\mu}}}}{\left( \pm i \sqrt{10} k \beta^{1 / 4} \sqrt{\mu} c_{1} e^{\mp i \frac{\sqrt{2 \sqrt{\beta} \gamma-i \sqrt{10} \alpha \sqrt{\mu}}}{\sqrt{10} k \beta^{1 / 4} \sqrt{\mu}}}+c_{2} \sqrt{2 \sqrt{\beta} \gamma-i \sqrt{10} \alpha \sqrt{\mu}}\right)^{2}}
$$

Simplifying the required exponential solution (3.23) into trigonometric function, we derive the solution of the Eq. (3.1) in the following:

$$
u(x, t)=\mp \frac{6 k \beta^{-3 / 4}(2 \sqrt{\beta} \gamma-i \sqrt{10} \alpha \sqrt{\mu})^{3 / 2} c_{1} c_{2}}{\left\{\sqrt{10} k \beta^{1 / 4} \sqrt{\mu} c_{1}(\cos (\vartheta) \mp i \sin (\vartheta)) \mp i c_{2} \sqrt{2 \sqrt{\beta}} \gamma-i \sqrt{10} \alpha \sqrt{\mu}(\cos (\vartheta) \pm i \sin (\vartheta))\right\}^{2}}
$$

where

$$
\vartheta=\xi \frac{\sqrt{2 \sqrt{\beta} \gamma-i \sqrt{10} \alpha \sqrt{\mu}}}{\sqrt{10} k \beta^{1 / 4} \sqrt{\mu}}=\frac{50 \sqrt{10} \beta \mu x+\left(8 \sqrt{10} \beta \gamma^{2}-i 30 \alpha \sqrt{\beta} \gamma \sqrt{\mu}+5 \sqrt{10} \alpha^{2} \mu\right) t}{1000 \beta^{5 / 4} \mu^{3 / 2}(2 \sqrt{\beta} \gamma-i \sqrt{10} \alpha \sqrt{\mu})^{-1 / 2}} .
$$

Thus, we get the exact solution (3.24) of the generalized fifth-order $\mathrm{KdV}$ equation (3.1). But $c_{1}$ and $c_{2}$ are arbitrary constants, so, one may arbitrarily pick their values. Therefore, if we choose $c_{1}=\sqrt{2 \sqrt{\beta}} \gamma-i \sqrt{10} \alpha \sqrt{\mu}$ and $c_{2}=-\sqrt{10} k \beta^{1 / 4} \sqrt{\mu}$, then the solitary wave solution (3.24) becomes

$$
u_{5}(x, t)=\frac{3\left(2 \sqrt{10} \beta \gamma^{2}-i 20 \alpha \sqrt{\beta} \gamma \sqrt{\mu}-5 \sqrt{10} \alpha^{2} \mu\right)}{5 \beta(i 2 \sqrt{\beta} \gamma+\sqrt{10} \alpha \sqrt{\mu}) \sqrt{\mu}\left\{1-\sin \left(\frac{\vartheta}{2}\right)\right\}} .
$$

Again, if we choose $c_{1}=\sqrt{2 \sqrt{\beta} \gamma-i \sqrt{10} \alpha \sqrt{\mu}}$ and $c_{2}=\sqrt{10} k \beta^{1 / 4} \sqrt{\mu}$, then from (3.24), we obtain the following solitary wave solution:

$$
u_{6}(x, t)=\frac{3\left(2 \sqrt{10} \beta \gamma^{2}-i 20 \alpha \sqrt{\beta} \gamma \sqrt{\mu}-5 \sqrt{10} \alpha^{2} \mu\right)}{5 \beta(i 2 \sqrt{\beta} \gamma+\sqrt{10} \alpha \sqrt{\mu}) \sqrt{\mu}\left\{1+\sin \left(\frac{\vartheta}{2}\right)\right\}}
$$

On the other hand, if we take $c_{1}=\sqrt{2 \sqrt{\beta}} \gamma-i \sqrt{10} \alpha \sqrt{\mu}$ and $c_{2}= \pm i \sqrt{10} k \beta^{1 / 4} \sqrt{\mu}$, then from (3.24), we get the solution in the form:

$$
u_{7}(x, t)=-\frac{i 3(\sqrt{10} \sqrt{\beta} \gamma-i 5 \alpha \sqrt{\mu})}{5 \beta \sqrt{\mu}\left\{1+\cos \left(\frac{\vartheta}{2}\right)\right\}} .
$$

Also if $\quad c_{1}=\sqrt{2 \sqrt{\beta} \gamma-i \sqrt{10} \alpha \sqrt{\mu}} \quad$ and $c_{2}=\mp i \sqrt{10} k \beta^{1 / 4} \sqrt{\mu}$, then the solitary wave solution (3.24) can be written in the form:

$$
u_{8}(x, t)=\frac{3(i \sqrt{10} \sqrt{\beta} \gamma+5 \alpha \sqrt{\mu})}{5 \beta \sqrt{\mu}\left\{-1+\cos \left(\frac{\vartheta}{2}\right)\right\}} .
$$

But, since $c_{1}$ and $c_{2}$ are arbitrary constants for other choices of $c_{1}$ and $c_{2}$, we might obtain much new and more general exact solutions to Eq. (3.1) by the MSE method without any aid of symbolic computation software. The major advantage of the MSE method is that the calculations are not sophisticated and easy to control. It is not required any computer algebra system to facilitate the calculations as it take to the Exp-function method, the $\left(G^{\prime} / G\right)$-expansion, the tanh-function method, the homotopy analysis method etc. But the solutions obtained by the MSE method are equivalent to those solutions obtained by the above mentioned method.

Remark: Solutions (3.19)-(3.22) and (3.25)-(3.28) have been verified by putting them back into the original equation and found correct.

\section{Explanations and Physical Interpretations of the Solutions}

In this section, we will depict the graph and signify the obtained solutions to the generalized fifth-order $\mathrm{KdV}$ equation. The solutions (3.19) to (3.22) are represents the periodic solutions. These solutions are traveling wave solutions that are all periodic bell shape but different, such that Fig. 1 shows the bell shape of the solitons (3.19) to (3.20) and Fig. 2 drawn the bell shape solitons (3.21) to (3.22) are respectively for $\alpha=\gamma=\mu=k=1$ and $\beta=-1$ within $-10 \leq x, t \leq 10$. Solitons are solitary waves with resilient scattering property. On the other hand, Fig. 3 and Fig. 4 are plotted the periodic solitons (3.25)-(3.26) and (3.27)-(3.28) respectively for $\alpha=\beta=-1$ and $\gamma=\mu=k=1$ 
within $-10 \leq x, t \leq 10$. Here, the Figures 1 to 2 and the figures 3 to 4 are sketch of same type but all are different.
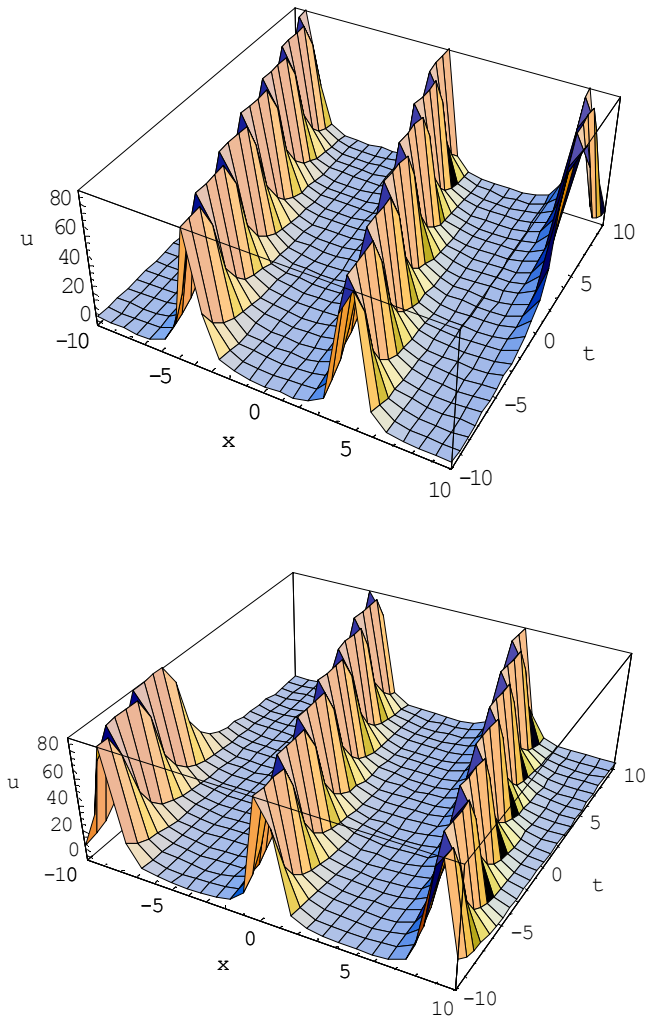

Fig. 1. Bell shape multi-solitons of solutions (3.19) and (3.20) to the generalized fifth-order KdV equation (3.1).
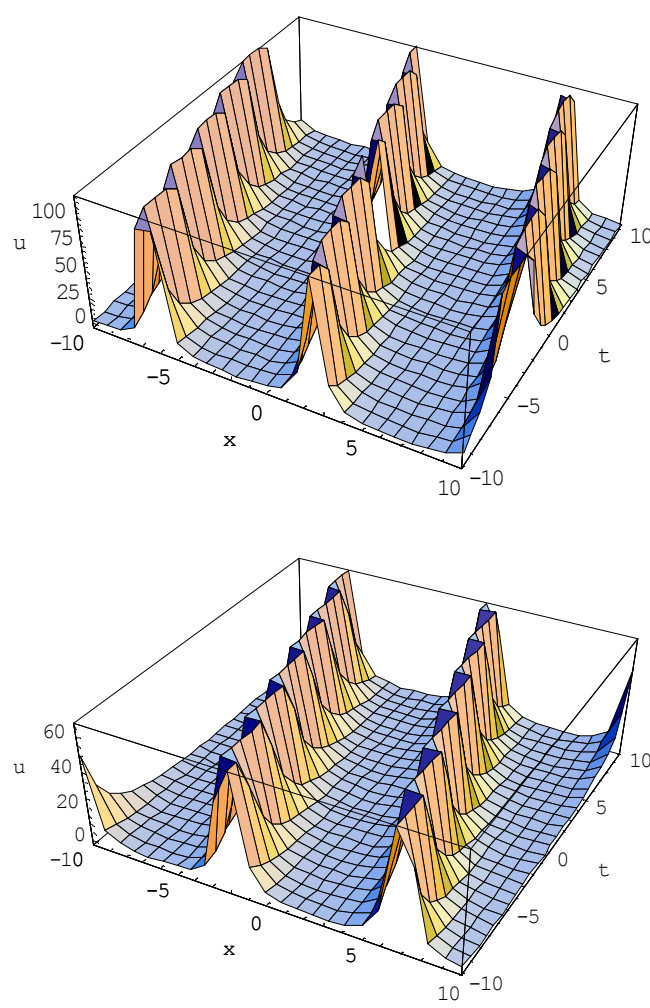

Fig. 2. Bell shape multi-solitons of solutions (3.21) and (3.22) to the generalized fifth-order KdV equation (3.1).
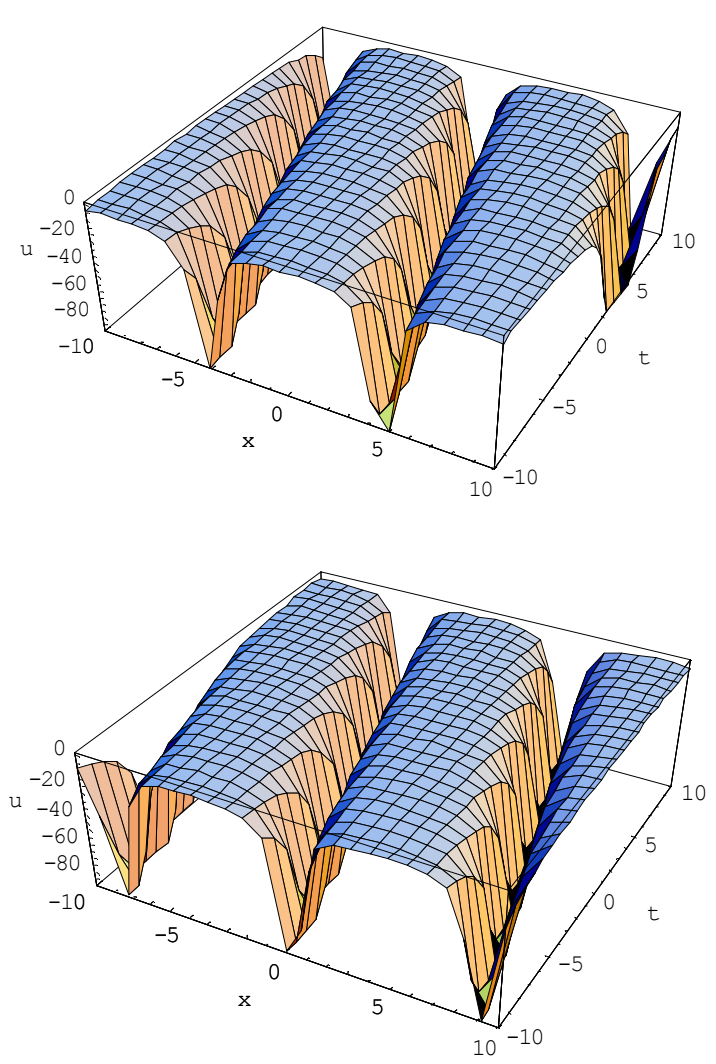

Fig. 3. Dark solitons of solutions (3.25) and (3.26) to the generalized fifthorder KdV equation (3.1).
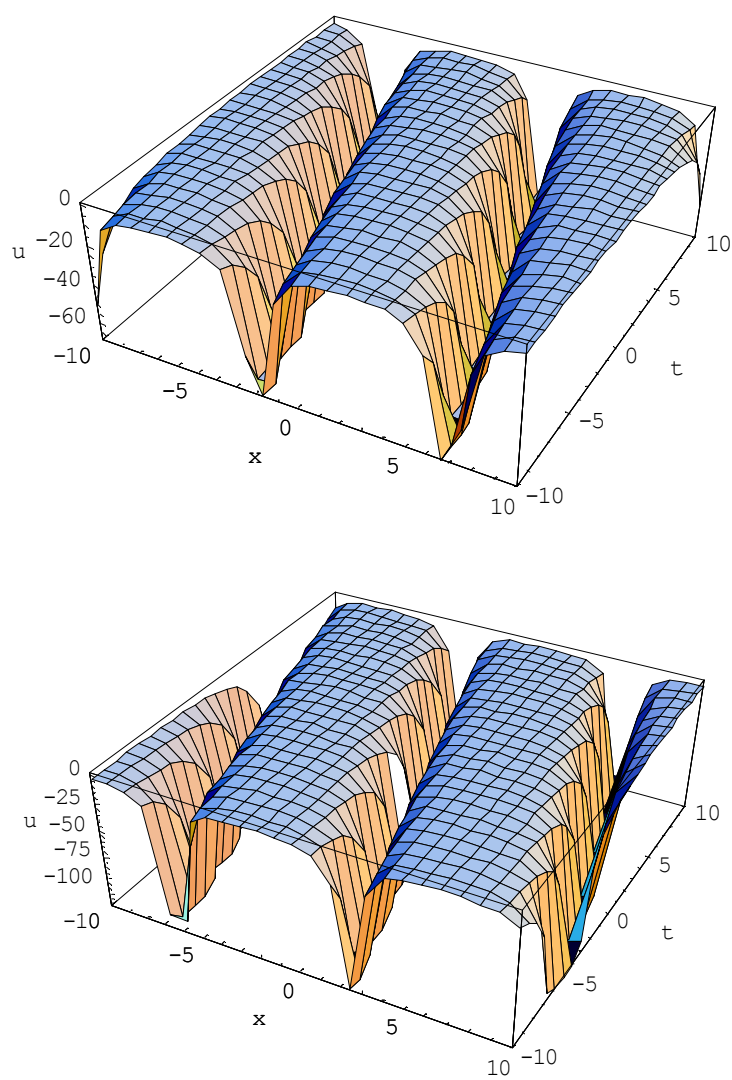

Fig. 4. Dark solitons of solutions (3.27) and (3.28) to the generalized fifthorder KdV equation. 


\section{Conclusions}

If the balance number is greater than one, in general the MSE method does not provide any solution. For this case, we have established a procedure in order to implement the MSE method to solve NLEEs for balance number two. If the solution of $s(\xi)$ consists of polynomial of the wave variable $\xi$, it will not be the solitary wave solution, since it does not meet the condition $|u| \rightarrow 0$ as $\xi \rightarrow \pm \infty$ for solitary wave solution. In this case, each coefficient of the polynomial must be zero. This constraint is crucial to solve NLEEs for higher balance number. By using this achieved process, we have solved the generalized fifth-order $\mathrm{KdV}$ equation and found some new traveling wave solutions. When the parameters receive special values, solitary wave solutions are derived from the exact solutions. We analyze the solitary wave properties of the solutions via the graphs.

\section{References}

[1] M.J. Ablowitz and P.A. Clarkson, "Soliton, nonlinear evolution equations and inverse scattering", Cambridge University Press, New York, 1991.

[2] R. Hirota, "The direct method in soliton theory", Cambridge University Press, Cambridge, 2004.

[3] C. Rogers and W.F. Shadwick, "Backlund transformations and their applications", Vol. 161 of Mathematics in Science and Engineering, Academic Press, New York, USA, 1982.

[4] L. Jianming, D. Jie and Y. Wenjun, "Backlund transformation and new exact solutions of the Sharma-Tasso-Olver equation", Abstract Appl. Analysis, 2011 (2011) Article ID 935710, 8 pages.

[5] V.B. Matveev and M.A. Salle, "Darboux transformation and solitons”, Springer, Berlin, 1991.

[6] J. Weiss, M. Tabor and G. Carnevale, "The Painlevé property for partial differential equations", J. Math. Phys., 24 (1982),pp. 522-526.

[7] A.M. Wazwaz, "Partial Differential equations: Method and Applications", Taylor and Francis, 2002.

[8] M.A. Helal and M.S. Mehana, "A comparison between two different methods for solving Boussinesq-Burgers equation", Chaos, SolitonsFract., 28 (2006),pp. 320-326.

[9] D.D. Ganji, “The application of He's homotopy perturbation method to nonlinear equations arising in heat transfer", Phys. Lett. A, 355 (2006), pp. 137-141.

[10] D.D. Ganji, G.A. Afrouzi and R.A. Talarposhti, "Application of variational iteration method and homotopy perturbation method for nonlinear heat diffusion and heat transfer equations", Phys. Lett. A, 368 (2007), pp. 450-457.

[11] G. Xu, "An elliptic equation method and its applications in nonlinear evolution equations", Chaos, SolitonsFract., 29 (2006), pp. 942-947.

[12] E. Yusufoglu and A. Bekir, "Exact solution of coupled nonlinear evolution equations", Chaos, solitonsFract., 37 (2008), pp. 842-848.
[13] T.L. Bock and M.D. Kruskal, "A two-parameter Miura transformation of the Benjamin-Ono equation", Phys. Lett. A, 74 (1979),pp. 173-176.

[14] A.M. Wazwaz, A sine-cosine method for handle nonlinear wave equations, Appl. Math. Comput. Modeling, 40 (2004) 499-508.

[15] E. Yusufoglu, and A. Bekir, "Solitons and periodic solutions of coupled nonlinear evolution equations by using sine-cosine method", Int. J. Comput. Math., 83 (12) (2006), pp. 915-924.

[16] M. Wang, "Solitary wave solutions for variant Boussinesq equations", Phy. Lett. A, 199 (1995),pp. 169-172.

[17] W. Malfliet and W. Hereman, "The tanh method II: Perturbation technique for conservative systems", Phys. Scr., 54 (1996), pp. 563-569.

[18] H.A. Nassar, M.A. Abdel-Razek and A.K. Seddeek, "Expanding the tanh-function method for solving nonlinear equations", Appl. Math., 2 (2011),pp. 1096-1104.

[19] A.J.M. Jawad, M.D. Petkovic, P. Laketa and A. Biswas, "Dynamics of shallow water waves with Boussinesq equation", ScientiaIranica, Trans. B: Mech. Engr., 20(1) (2013), pp. 179-184.

[20] M.A. Abdou, "The extended tanh method and its applications for solving nonlinear physical models", Appl. Math. Comput., 190 (1) (2007),pp. 988-996.

[21] N. Taghizadeh and M. Mirzazadeh, "The first integral method to some complex nonlinear partial differential equations", J. Comput. Appl. Math., 235 (2011),pp. 4871-4877.

[22] M.L. Wang and X.Z. Li, "Extended F-expansion method and periodic wave solutions for the generalized Zakharov equations", Phys. Lett. A, 343 (2005), pp. 48-54.

[23] Sirendaoreji, "Auxiliary equation method and new solutions of Klein-Gordon equations", Chaos, SolitionsFract., 31 (2007), pp. 943-950.

[24] A.L. Guo and J. Lin, "Exact solutions of (2+1)-dimensional HNLS equation", Commun. Theor. Phys., 54 (2010), pp. 401406.

[25] S.T. Mohyud-Din, , M.A. Noor and K.I. Noor, "Modified variational iteration method for solving sine-Gordon equations", World Appl. Sci. J., 6 (7) (2009), pp. 999-1004.

[26] H. Triki, A. Chowdhury and A. Biswas, "Solitary wave and shock wave solutions of the variants of Boussinesq equation", U.P.B. Sci. Bull., Series A, 75(4) (2013),pp. 39-52.

[27] H. Triki, A.H. Kara and A. Biswas, "Domain walls to Boussinesq type equations in (2+1)-dimensions", Indian $\mathrm{J}$. Phys., 88(7) (2014),pp. 751-755.

[28] J.H. He and X.H. Wu, "Exp-function method for nonlinear wave equations", Chaos, SolitonsFract., 30 (2006),pp. 700708.

[29] H. Naher, A.F. Abdullah and M.A. Akbar, "New traveling wave solutions of the higher dimensional nonlinear partial differential equation by the Exp-function method", J. Appl. Math.,2012 (2012) Article ID 575387, 14 pages.

[30] M. Wang, X. Li and J. Zhang, "The $\left(G^{\prime} / G\right)$-expansion method and traveling wave solutions of nonlinear evolution equations in mathematical physics", Phys. Lett. A, 372 (2008), pp. 417-423. 
[31] J. Zhang, F. Jiang and X. Zhao, “An improved $\left(G^{\prime} / G\right)$ expansion method for solving nonlinear evolution equations", Inter. J. Comput. Math., 87(8) (2010),pp. 1716-1725.

[32] J. Feng, W. Li and Q. Wan, "Using $\left(G^{\prime} / G\right)$-expansion method to seek the traveling wave solution of KolmogorovPetrovskii-Piskunov equation", Appl. Math. Comput., 217 (2011),pp. 5860-5865.

[33] M.A. Akbar, N.H.M. Ali and E.M.E. Zayed, "Abundant exact traveling wave solutions of the generalized Bretherton equation via $\left(G^{\prime} / G\right)$-expansion method", Commun. Theor. Phys., 57 (2012),pp. 173-178.

[34] R. Abazari, "The $\left(G^{\prime} / G\right)$-expansion method for Tziteica type nonlinear evolution equations", Math. Comput. Modelling, 52 (2010), pp. 1834-1845.

[35] M.A. Akbar, N.H.M. Ali and S.T. Mohyud-Din, "Further exact traveling wave solutions to the $(2+1)$-dimensional Boussinesq and Kadomtsev-Petviashvili equation", J. Comput. Analysis Appl., 15 (3) (2013),pp. 557-571.

[36] A.J.M. Jawad, M.D. Petkovic and A. Biswas, "Modified simple equation method for nonlinear evolution equations", Appl. Math. Comput., 217 (2010), pp. 869-877.

[37] E.M.E. Zayed and S.A.H. Ibrahim, "Exact solutions of nonlinear evolution equations in mathematical physics using the modified simple equation method", Chin. Phys. Lett., 29(6) (2012), 060201.
[38] K. Khan, M.A. Akbar and M.N. Alam, "Traveling wave solutions of the nonlinear Drinfel'd-Sokolov-Wilson equation and modified Benjamin-Bona-Mahony equations", J. Egyptian Math. Soc., 21 (2013),pp. 233-240.

[39] K. Khan and M. A. Akbar, "Exact and solitary wave solutions for the Tzitzeica-Dodd-Bullough and the modified Boussinesq-Zakharov-Kuznetsov equations using the modified simple equation method", Ain Shams Engr. J., 4 (2013), pp. 903-909.

[40] K. Khan and M.A. Akbar, "Traveling wave solutions of some coupled nonlinear evolution equations", ISRN Math. Phys., 2013 (2013) Art. ID 685736, 8 pages.

[41] K. Khan and M.A. Akbar, "Application of $\exp (-\phi(\xi))$ expansion method to find the exact solutions of modified Benjamin-Bona-Mahony equation", World Appl. Sci. J., 24(10) (2013),pp. 1373-1377.

[42] M.G. Hafez, M.N. Alam and M.A. Akbar, "Traveling wave solutions for some important coupled nonlinear physical models via the coupled Higgs equation and the Maccari system”, J. King Saud Univ.-Sci., 27 (2015), pp.105-112.

[43] M.A. Salam, "Traveling wave solution of modified Liouville equation by means of modified simple equation method", ISRN Appl. Math., Vol. 2012, Article ID 565247, 4 pages.

[44] E.M.E. Zayed and A.H. Arnous, "Exact traveling wave solutions of nonlinear PDEs in mathematical physics using the modified simple equation method", Appl. Appl. Math.: An Int. J., 8(2) (2013), pp. 553-572. 